\title{
Les transformations des services urbains à Salvador de Bahia
}

Tania Fischer, Florence Heber, Dominique Lorrain, Elvia Fadul

\section{Citer ce document / Cite this document :}

Fischer Tania, Heber Florence, Lorrain Dominique, Fadul Elvia. Les transformations des services urbains à Salvador de Bahia. In: Flux, n¹0, 1992. pp. 32-40;

doi : https://doi.org/10.3406/flux.1992.917

https://www.persee.fr/doc/flux_1154-2721_1992_num_8_10_917

Fichier pdf généré le 12/05/2018 


\begin{abstract}
Tania FISCHER and Florence HEBER, The transformation of urban services in Salvador de Bahia. This article discusses how the Brazilian city of Salvador de Bahia has dealt with the problems of managing urban technical networks. The former system of public administration, a legacy from the period right after the war, proved to be incapable of dealing with a situation which rapidly evolved over the past twenty years. The city has become more spread out, the population has become more polarized, and the structure of the city is a combination of networked zones with completely unzoned, illegally developed areas. The city has thus turned to private companies. The authors of the article describe the changes in three key sectors: water utilities, garbage removal and transportation. They show that the public authories must create new, stable relationships with their partners and with customers, which touches on problems of the level of responsibility, forms of controls, and payment.
\end{abstract}

\title{
Résumé
}

Tania FISCHER et Florence HEBER, Les transformations des services urbains à Salvador de Bahia. Cet article montre comment une ville brésilienne se trouve confrontée à des problèmes de gestion des réseaux techniques urbains. L'ancien système d'administration publique hérité de l'après guerre ne parvient pas à faire face à une situation qui évolue très rapidement depuis vingt ans. La ville s'est étendue, sa population s'est polarisée et sa structure urbaine combine des zones maillées avec des zones de développement illégal. La municipalité se tourne alors vers l'entreprise privée. Les auteurs décrivent les changements dans trois secteurs clef : l'eau, les transports et les déchets. Ils montrent que les autorités publiques doivent créer avec leurs partenaires et les usagers de nouvelles relations stables, ce qui touche au niveau de l'autorité responsable, aux formes du contrôle, au mode de rémunération. 
FLUX 10 Octobre - Décembre 1992

Illustration non autorisée à la diffusion

FIGLRE: 1 : Evolution de l'occupation urbaine de Salvador - 1940 (source : Bahia Conder 1985) 


\title{
LES TRANSFORMATIONS DES SERVICES
}

\author{
URBAINS À SALVADOR DE BAHIA
}

TANIA FISCHER \& FLORENCE HEBER

Tania FISCHER dirige le troisième cycle d'administration de l'Université Fédérale de Babia et joue un rôle important dans l'animation des milieux de recherche au Brésil.

Florence HEBER a fait un mastère sur le service des déchets urbains à Salvador après un séjour de quelques mois au Laboratoire Techniques-TerritoiresSociétés (ENPC, Paris-XII). Elle travaille à l'organisme de planification de l'Etat de Babia.
E PROCESSUS RAPIDE de transformation de la gestion des $\mathcal{L}$ services urbains à Salvador, capitale de l'Etat de Bahia au Brésil, est nettement perceptible, comme dans d'autres villes brésiliennes. Parallèlement aux innovations techniques, de nouvelles expériences de gestion remettent en cause le rapport public-privé, les formes de financement, les rôles des acteurs - gestionnaires publics, syndicats et mouvements associatifs. L'analyse de ces processus ne peut se limiter à une simple approche sectorielle de ce qui se passe au moment présent. Il faut à la fois rechercher dans le passé les explications du présent, et voir ces services d'une façon dynamique, comme un réseau de relations construit de plusieurs fils et noeuds : en transformation constante.

\section{L'ORGANISATION DE L'ESPACE}

Salvador, capitale de l'Etat de Bahia, fut la première capitale du Brésil. A l'heure actuelle, c'est une métropole moderne, à forte vocation touristique dont le développement a connu un très grand essor pendant les années 1970. Cette modernité cohabite avec une pauvreté résultant d'une très forte disparité des revenus. La ville s'étend sur une surface de $324 \mathrm{~km}^{2}$. L'ancien * centre historique * considéré par les historiens comme un patrimoine de l'humanité se trouve en très mauvais état de conservation physique.

Depuis sa création, la ville s'est toujours ajustée aux conditions des cycles économiques par lesquels elle est passée. Ils ont finit par modeler une image urbaine spécifique à chaque période. La concentration autour de Salvador qui démarre dès l'époque coloniale établit son rôle de centre de distribution de marchandises et de services pour toute la région. Dans cette phase, la ville se réduit spatialement à un noyau central dont les limites ne sont pas modifićes jusqu'à la moitié du XXème siècle. Au début de ce siècle, l'expansion urbaine s'est visiblement intensifiće, notamment sur le littoral. 
Entre 1950 et 1965, se sont développées des zones de peuplement illégales à l'origine ${ }^{1}$ qui concentraicnt des populations à bas revenu : "Nordeste de Amaralina ", "Alagados " au bord de la mer. Le développement de la lisière maritime s'est poursuivi. Pendant cette période, Salvador élargit son territoire, décentralise ses activités économiques de façon à soutenir son processus d'industrialisation et donne naissance à une région urbaine. De nouveaux foyers urbains se développent le long des voiries sortant de la ville où se concentrent les zones industrielles de la Région Métropolitaine.

La stratégie d'aménagement spatial adoptée à l'époque envisageait la préservation du patrimoine historico-culturel de la ville qui devrait rester comme une base d'appui. Effectivement, on a réussi de ce côté là, puisque Salvador est devenue la ville dortoir de la population employée dans les 56 entreprises du Pôle Pétrochimique de Camaçari. Cela n'empêche pas d'avoir une forte concentration de la population active (44\%) dans le marché informel du travail.

A partir de 1970 , on remarque la présence très forte des "invasions " dans le noyau de la ville qui avait à cette époque 1.007.195 habitants. Ies conditions de logement sont extrêmement précaires pour $30 \%$ de la population. Il faut ajouter que presque $50 \%$ des gens ont un revenu mensuel inférieur à 100 dollars. Dans les nouveaux quartiers, une nouvelle architecture, osée et créative, cohabite à côté des "invasoes", ensembles de logements précaires construits sur des terrains occupés de façon illégale. Il y a là une remarquable tension entre la ville moderne, légale, touristique, et la ville construite avec des décombres, à peine tolérée et qui, petit à petit, est déplacée vers la périphérie. De ce fait, les limites urbaines s'élargissent.

L'incapacité de la mairic à réduire ces tensions est visible et ce vide dans l'exercice du pouvoir va engendrer des réactions qui sont à l'origine des mouvements associatifs articulés autour d'un axe de revendications pour de meilleures conditions de vie urbaine : infrastructures, équipements, services urbains. Ces mouvements sont structurés sur les associations de " moradores" (citadins - riverains) qui se forment à partir des liens associatifs tels que les pratiques religicuses, les activités sportives, culturelles ou artistiques; il faut aussi considérer les liens parentaux, de voisinage et d'amitić.
Le gouvernement local et le mouvement associatif constituent les deux pôles d'expression de ce qu'on appelle le pouvoir local. Quand on parle de * local ", on n'est limité ni à la notion de quartier ou d'arrondissement, ni à la notion de ville. L'univers d'analyse est plus large et plus abstrait. Il peut être rapporté, soit au quartier et à la municipalité, soit à ses limites périphériques, mais pas nécessairement. Parler de * local " signifie parler de l'ensemble de réseaux qui s'articulent et se superposent, autant les réseaux sociaux que les réseaux techniques. Les premiers recouvrant l'ensemble des rapports de coopération ou de conflits d'intérêts, de ressources, d'équipements et de valeurs, et cela dans un espace dont les limites sont définies par cet ensemble de réseaux.

Dans ce sens, les services urbains intègrent aussi ce réseau dans un processus qui présente des dimensions multiples d'analyse allant des plus visibles aux moins visibles, à savoir : les dimensions physiques, économiques, institutionnelles et sociales.

\section{LA GESTION DES SERVICES URBAINS}

La ville s'est donc développée en élargissant sa maille urbaine, ce qui concerne l'espace et le territoire effectivement occupés. C'est peut-être ce modèle de développement spatial centré sur les extrémités de ses limites géographiques, laissant au milieu de grandes aires inoccupées ou à faible densité, qui explique la concentration de services urbains dans les aires centrales de la ville pendant que dans d'autres aires très vastes, l'absence de ces services est flagrante.

L'occupation de l'espace urbain à Salvador s'organise en deux formes très visibles. D'une part, une occupation "institutionalisée ", la ville légale sur une trame assainie, ordonnée, respectant les critères et les normes de construction et d'urbanisation de la municipalité. Cette occupation s'étale dans des espaces considérés comme adaptés à la construction, sur des zones où le relief n'est pas trop accentué (sur les crêtes et les versants) ou dans les vallées à proximité de la mer. Cette forme urbaine ne représente plus que $30 \%$ du territoire de la ville. D'autre part, on voit une autre forme d'occupation 
Fischer \& Heber - Services à Salvador de Bahia

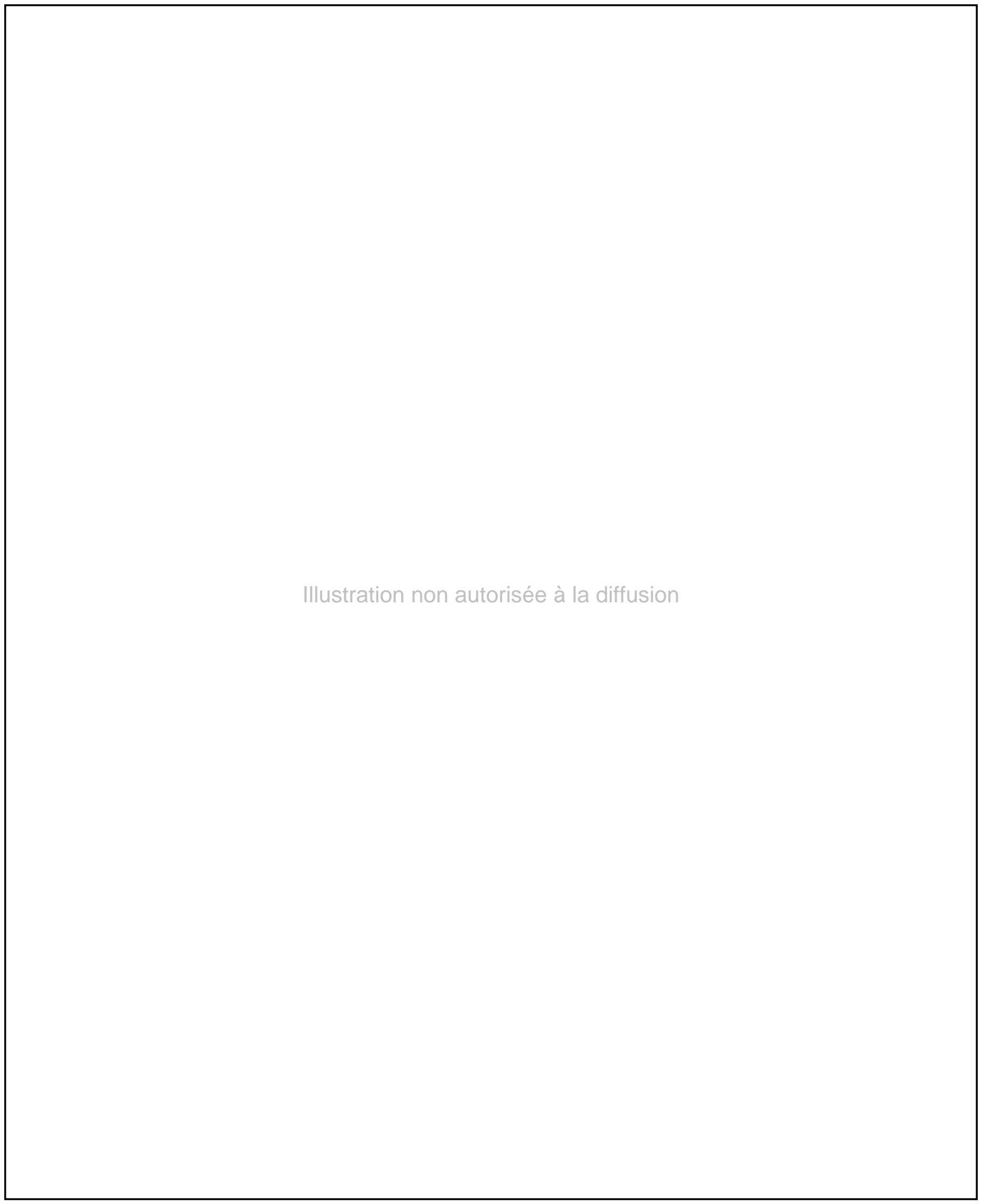

FICI:RE 2 : Forolution de l'occupation urbaine de Salvador - 1970 (source : Bahia Conder 1985) 
désordonnée, en croissance, entièrement * spontanée ", caractérisée par une construction accélérée de logements aussi bien que par une concentration démographique intense.

De nos jours, avec 2.056.000 habitants, la ville reste dans ce modèle d'expansion urbaine caractérisé par une occupation de son noyau central par la population à bas revenu, à côté d'une intense occupation du littoral dans le secteur nord par des populations à niveaux de revenu plus élevés, et aussi par des commerces et des services sophistiqués.

Depuis la décennie soixante-dix, les services urbains de base ont reçu plus d'attention du gouvernement local. Cela s'est manifesté au plan institutionnel par la création d'entreprises publiques suivant le modèle de gestion adopté alors par le gouvernement fédéral : planification et entreprise publiques. Au niveau local, ces entreprises sont devenues les responsables de l'organisation et de la production de certains services urbains. L'intention du gouvernement était de rendre possible dans certains secteurs d'activité l'utilisation d'outils semblables à ceux qui existent dans le secteur privé, une plus grande flexibilité au niveau de la gestion, l'autonomie financière et une certaine indépendance vis-à-vis de l'administration municipale stricto sensu.

Le résultat actuel diffère beaucoup de la proposition originale. Assujetties à la logique de gestion des organes de l'administration directe, les entreprises publiques de services urbains ont accumulé au long des années un grand excédent de personnel, des dettes financières et des méthodes de gestion contradictoires avec une logique d'efficacité et de productivité. Pendant les années du régime autoritaire, quand les informations sur les procédures de l'administration publique étaient pratiquement inexistantes, certaines se sont transformées dans des bastions d'influence des élus locaux. Cette période a donc donné lieu à un type de performance con. duisant à caractériser l'administration publique comme étant plus une "administration " qu'une organisation au service du public.

Toutefois, il est juste d'affirmer que l'offre des services a été amplifiée pendant cette période, à Salvador comme dans les autres villes du pays, car c'était nécessaire pour viabiliser le processus productif ; dans une ville spatialement élargie comme Salvador, le transport public jouc un rôle fondamental dans l'activité économique.

Dans la situation actuclle, il faut aussi prendre en compte ce qui relève des modes de vie. Ia ville s'est développée; elle est devenue très complexe et a incorporé des modes de vie incompatibles avec des solutions individualisées. Il y a vingt ans, on trouvait normale la construction de systèmes de captation d'eau par des puits, on voyait des solutions d'as. sainissement autonome avec des fosses individuelles, ou même des systèmes particuliers d'élimination des déchets des ménages tels que brûler les ordures ou enterrer les résidus dans le jardin. Aujourd'hui ce genre de solution est très rare.

En ce qui concerne le financement, le transport en commun et l'assainissement sont couverts par les tarifs. La propreté urbaine a pour principale source de financement le budget municipal (impôts sur le foncier). Ces tarifs sont en général très élevés par rapport au niveau de revenu de la population, ce qui fait qu'une partie considérable de celle-ci ne s'inclut pas dans la catégoric d'usager permanent. Dans le cas des transports en commun, l'utilisation d'autres alternatives en dehors du système formel est quelque chose de normal.

Essayant de redéfinir certains réseaux de services et de maîtriser les problèmes de taux de couverture et de qualité, les organismes responsables de ces activités sont en ce moment en train de développer divers projets et plans d'intervention.

\section{NOUVEAUX MODÈLES D'ORGANISATION DES SERVICES}

Actuellement, on assiste à Salvador au développement d'une série de projets et de plans gouvernementaux qui visent à la mise en place de transformations significatives des modes de gestion, d'organisation et de production des services urbains, notamment dans les services considérés comme fondamentaux - l'eau, les déchets et les transports urbains. Quelles sont les bases de ces propositions et quels sont leurs objectifs principaux? 
Fischer \& Heber - Services à Salvador de Bahia

Illustration non autorisée à la diffusion

FIGLRE: 3 : Fvolution de loccupation urbaine de Salvador - 198.3 (source : Bahia Conder 1985) 
La redéfinition des modes de gestion des services semble être liée au besoin d'établir des paramètres et des mécanismes orientés vers des résultats spécifiques et vers la quête d'une plus grande efficacité dans l'offre du service. Pour cela, le secteur privé est engagé en tant qu'acteur " naturellement " porteur des idéaux de productivité et d'efficacité. Toutefois, les formes de contrat et l'étendue de la participation des entreprises privées dans ces services varient.

Dans le cas du service de l'eau, la proposition de l'entreprise publique chargée de cette activité est de partager l'exploitation du barrage de Pedra do Cavalo, ${ }^{2}$ située à environ $120 \mathrm{~km}$ de Salvador. Ie sectcur public, par un contrat de prestation de service, déléguerait la responsabilité des opérations de captation, de traitement, de contrôle, et d'adduction de l'eau traitée de la principale station de traitement d'eau, au secteur privé. Les arguments pour une telle politique sont fondés sur la perspective de réduction des coûts d'exploitation de Pedra do Cavalo, à travers la gestion du barrage par le secteur privé qui sera rémunéré par quantité de $\mathrm{m}^{3}$ d'eau traitée produite.

Cette initiative s'avère très polémique et se heurte à des résistances, notamment de la part du syndicat des ouvriers de l'eau et de l'assainissement, qui se sentent menacés par la perspective du chômage. Pourtant, compte tenu des difficultés de formation de personnel qualifié, il est probable que l'entreprise privée embauche des agents provenant de l'entreprise publique. Il faut aussi souligner que l'intervention du secteur privé dans ce service s'é. tend depuis quelques temps déjà à diverses activités. A titre d'exemple, nous pouvons citer la construction d'usines, de barrages, la fourniture d'équipements et l'implantation de réseaux d'eau. $\mathrm{Au}$ niveau de la gestion, des entreprises privées agissent dans des secteurs commerciaux, exécutent des tâches telles que des coupures d'approvisionnement et les branchements, l'installation et l'étalonnage des compteurs, la réparation de petites fuites, la délivrance des factures, l'inspection, la location de véhicules, la nettoiement, le suivi et le contrôle des travaux d'égouts entre autres.

De fait, la question actuelle porte sur la proportion de la participation du privé, toujours perçue par les opposants d'une telle politique comme le premier pas vers la privatisation totale du service. L'argument principal de ces opposants est basé sur l'idée qu'avec un contrat général de délégation ${ }^{3}$, l'entreprise publique pourrait perdre le contrôle de " son affaire ", en considérant que le secteur privé, par nature, possède des caractéristiques naturelles qui ne sont pas compatibles avec l'offre des " services essenticls .

Dans les transports urbains, les propositions sont d'une nature différente et se concentrent sur l'optimisation des trajets, sur la création de sites propres pour les autobus. sur l'informatisation du système en privilégiant le transport collectif.

Du point de vue de la régulation public-privé, la proposition vise à établir plus de rationalité dans l'organisation du réseau et à renforcer le contrôle des pouvoirs publics sur l'offre provenant des entreprises privées par l'intermédiaire du Secrétariat Municipal de Transport. Ia TRANSUR, entreprise publique, continuera de jouer son rôle d'élément régulateur du système en exploitant les lignes considérées comme déficitaires.

Les * contrats de permission " obéissent à de nouveaux critères, à savoir :

a) Ils sont accordés pour des lots identiques et non ligne par ligne;

b) au total seize entreprises se partagent onze lots et exploitent 387 lignes ;

c) les entreprises doivent modifier leurs lignes afin de réduire les parcours peu fréquentés;

d) le gouvernement local retiendra $30 \%$ de la recette journalière des entreprises exploitantes dans un Fond, le FUNIDESTRAN (Fond de Dévelop. pement du Transport Collectif de Salvador);

e) en fonction du respect du contrat signé - critères de productivité - l'entreprise exploitante pourra récupérer ces $30 \%$ de la recette.

Pour le mode de rémunération du service, ce nouveau modèle affirme un système de tarification unique, avec paiement par l'usager auprès de l'exploitant. Avec le mécanisme de retenue de $30 \%$ de la recette, l'autorité publique se dote d'un moyen de contrôle sur les entreprises privées exploitantes des lignes. Les critères de restitution des $30 \%$ sont établis sur la flotte de véhicules destince au transport, sur les kilomètres parcourus et sur le nombre de passagers transportés. Ie contrôle de la qualité des 
services effectivement offerts nécessitera la mise en oeuvre d'un système d'information dans les gares afin de vérifier tous les aspects qu'on vient de décrire.

Dans le service de propreté publique, un plan direc. teur $^{4}$ est en cours d'élaboration. Il indiquera un cadre institutionnel et financier plus adapté aux conditions techniques du système, en particulier l'utilisation d'une seule décharge contrôlée - la Décharge Centrale - pour la destination finale des résidus des communes de Salvador, Lauro de Freitas et Simoes Filho. Avant de démarrer ce plan, d'autres actions sont en cours dans la municipalité de Salvador. Pour la troisième fois en 20 ans, le secteur privé est engagé pour exécuter une partie du service au moyen d'un contrat de prestation de service. On ne connaît pas pour autant les critères de rémunération de l'entreprise. La proposition initiale de la LIMPURB (entreprise publique de propreté publique) était de partager environ $70 \%$ du territoire de la ville entre deux entreprises privées. Cette proposition n'a pas abouti entièrement, car aujourd'hui une seule entreprise se trouve chargée du nettoiement, du ramassage des déchets et de la gestion de la décharge existante ${ }^{5}$. Cette entreprise, la Paviter, filiale d'un des plus grands groupes de construction brésilien - OAS - assure $50 \%$ de la collecte, le reste étant assuré par la LIMPURB. Sa rémunération se fait par une sorte de marché d'entreprise, avec paiement par la collectivité pour une prestation de service; elle perçoit \$36 par tonne enlevéc de déchet pour l'activité ramassage; l'activité de dé. charge est rémunérée à $\$ 6$ par tonne de déchet déposé.

Par ailleurs, la Mairie essaie pour la troisième fois dans un même mandat d'instituer unc taxe sur les déchets, établie sur la valeur de location des immeubles ce qui provoque toujours plusicurs résistances sociales. La contestation repose sur le principe que cette taxe entraînera une double imposition, car "l'impôt sur le Foncier "6 payé par tous les propriétaires d'immeubles, inclut déjà une part relative à la prestation de certains services urbains dont la propreté publique. Cette proposition aussi se heurte à des difficultés d'ordre juridique car la Constitution Brésilienne de 1988 exclut la possibilité d'une taxation sur des services dont les coûts sont indivisibles pour l'ensemble de la population.

\section{CONCLUSION}

Ce n'est donc pas une tâche aisée de bien gérer et de faire fonctionner convenablement les services urbains dans une telle ville. Se conjuguent des facteurs techniques et socio-économiques. I'un point de vue technique, le relief et la forme d'occupation du sol rendent difficile la construction des infras. tructures et la gestion des équipements de base. De plus, les conditions de revenu d'une grande majorité de la population ont de sérieuses conséquences sur la capacité financière de la municipalité. Beaucoup de difficultés demeurent avant qu'une politique de "services pour tous" se mette en oeuvre. Si l'on considère l'ampleur des besoins dans une ville qui a grandi énormément pendant les vingt dernières années, dans un modèle d'occupation qui n'accepte plus des solutions individualisées pour une grande partie des services, il est crucial que les politiques de services urbains obéissent à une logique d'intégration.

Le modèle de gestion prédominant reste encore basé sur la structure institutionnelle et organisation. nelle de l'entreprise publique. Ce qui change, c'est premièrement le niveau de l'autorité responsable, dans le cas de l'eau et de l'assainissement, l'EMBASA (Empresa Babiana de Saneamento) se rattache au niveau de l'administration fédérale, deuxièmement, c'est le recours croissant au secteur privé.

(traduit par Elvia Fadul et adapté par Dominique I.orrain) 


\section{FLUX 10 Octobre - Décembre 1992}

NOTES

1. Ce phénomène d'occupation illégale et soudaine d'un terrain kinvasoes» est permanent dans la ville.

2. Il s'agit de la source principale d'approvisionnement des villes de Salvador et de Feira de Santana, Lème ville de l'état de Bahia tant par sa superficie que par ses recettes fiscales, située à $110 \mathrm{~km}$ de Salvador au Nord-Ouest.

3. Délégation ou «terciarizaçao» dans le débat politique au Brésil, ou le fait de confier à un tiers. Le contrat de délégation «contratos de permissaom.

4. Plan Directeur de Propreté Publique de la Région Métropolitaine de Salvador.

5. Décharge qui sera fermée lorsqu'on exploitera la décharge centrale.

6. IPTU - «Imposto Predial e Territorial Urbanom - la Taxe sur la Propriété Foncière Immobilière.

\section{RÉFÉRENCES}

BAHIA, GOVERNO DO ESTADO. Termos de Referência para Elaboração do Estudo de Impacto Ambiental e do Relatório de Impacto Ambiental do Aterro Sanitário centro. Conder. Salvador, 1991.

CAdernos do NúCleo de los graduAçĩo em ADMinistruçĩo. IV Seminario. Governo Local : Poder e Participação. UFBA, Salvador, 1990.

HEBER, Florence. " Gestão do Lixo em Salvador. "Escola de Administração da Universidade Federal da Bahia. Thèse présentée pour l'obtention du diplôme de "Mestre en Administration".

FISCHER, Tania. “ Réseaux sociaux, logement et citoyenneté publique à Salvador Bahia ", colloque sur le logement, Montréal, 7-12 juillet 1992.

LIMA, Luis Maia. Tratamento do Lixo. Hemus, São Paulo, 1987.

MATTEDI, MR et al. Salvador, o processo de Urbanizaçäo. CPE/OCEPLAN, Salvador, 1979.

PREFEITURA DE SAlVAdOR. Plano de Ocupação para a Area do Miolo de Salvador. SEPLAN, 1985. 